\title{
Inbaltsverzeiđnis.
}

1. Zubemeines. Seite

1. Geididtlides $\ldots \ldots \ldots \ldots \ldots \ldots \ldots \ldots \ldots \ldots \ldots \ldots \ldots \ldots \ldots \ldots \ldots \ldots \ldots \ldots$

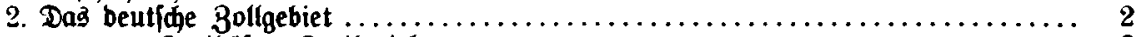

Freihäfen, Freibczirte $\ldots \ldots \ldots \ldots \ldots \ldots \ldots \ldots \ldots \ldots \ldots \ldots \ldots \ldots \ldots, 3$

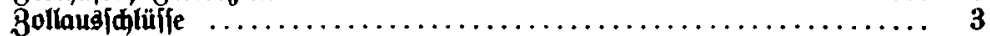

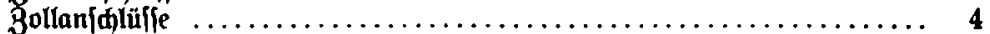

3. Der Brenzbezirt $\ldots \ldots \ldots \ldots \ldots \ldots \ldots \ldots \ldots \ldots \ldots \ldots \ldots \ldots \ldots \ldots \ldots \ldots$

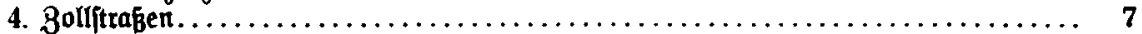

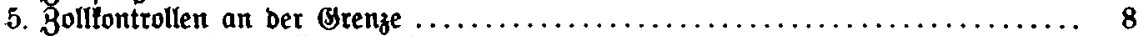

a) Beim $\mathfrak{x}_{\text {agenvertehr } \ldots \ldots \ldots \ldots \ldots \ldots \ldots \ldots \ldots \ldots \ldots \ldots \ldots \ldots} 8$

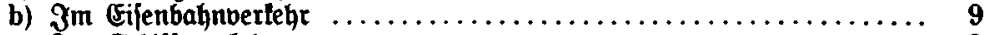

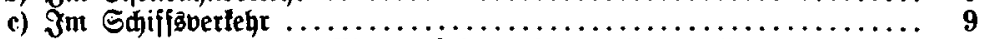

6. Die Berzollung ber eingegangenen Büter erfolgt auf bem Grenzzollamt $\ldots \ldots \ldots .9$

Bejonbere Erfiärung (ipezielle Deflaration) zum mareneingange $\ldots \ldots \ldots \ldots \ldots \ldots, 9$

7. Die Berzollung ber eingegangenen Süter erfolgt auf eittem in eren Fimte . . ... 12

Allgemeine Ertlärung (generelle Deflaration) zum $\mathfrak{B a r e n e i n g a n g e ~} \ldots \ldots \ldots \ldots \ldots 12$

8. Die \{pezielle Mevifion $\ldots \ldots \ldots \ldots \ldots \ldots \ldots \ldots \ldots \ldots \ldots \ldots \ldots \ldots \ldots \ldots \ldots \ldots$

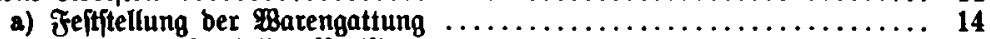

Erleidterungen bei ber fpeziellen Rebifion $\ldots \ldots \ldots \ldots \ldots \ldots \ldots \ldots \ldots \ldots \ldots \ldots \ldots \ldots$

b) Ermittelung bes Gerwiqtes $\ldots \ldots \ldots \ldots \ldots \ldots \ldots \ldots \ldots \ldots \ldots \ldots \ldots \ldots$

9. Die Entridtung bes Bollbetrages $\ldots \ldots \ldots \ldots \ldots \ldots \ldots \ldots \ldots \ldots \ldots \ldots \ldots \ldots \ldots \ldots$

a) Unmittelbar nad ber Bollabfertigung $\ldots \ldots \ldots \ldots \ldots \ldots \ldots \ldots \ldots \ldots 18$

b) Rad erjolgter Stunbung $\ldots \ldots \ldots \ldots \ldots \ldots \ldots \ldots \ldots \ldots \ldots \ldots \ldots \ldots \ldots$

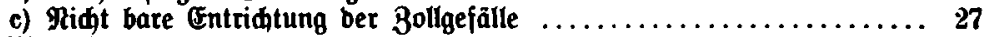

10. Bollfreie $\mathfrak{R i e b e r l a g e n} \ldots \ldots \ldots \ldots \ldots \ldots \ldots \ldots \ldots \ldots \ldots \ldots \ldots \ldots \ldots \ldots \ldots \ldots \ldots \ldots$

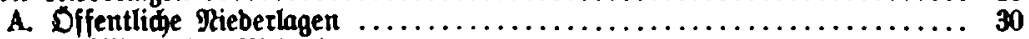

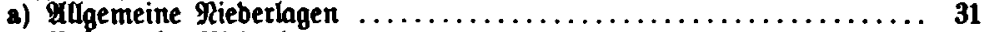

b) Pej

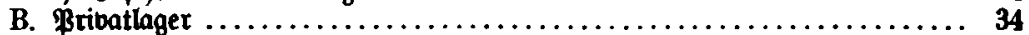

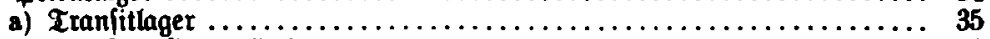

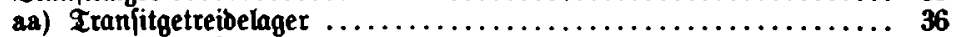

Peine ranfitgetreibelager $\ldots \ldots \ldots \ldots \ldots \ldots \ldots \ldots \ldots \ldots \ldots \ldots \ldots$

(Semijgte Iranjitgetreibelager $\ldots \ldots \ldots \ldots \ldots \ldots \ldots \ldots \ldots \ldots \ldots, 42$

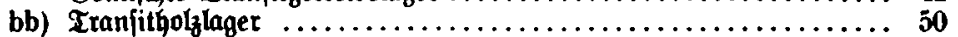

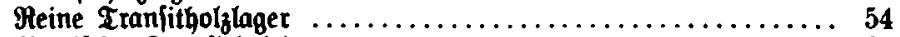

Gemijate Itanfitholzlager ...................... 61

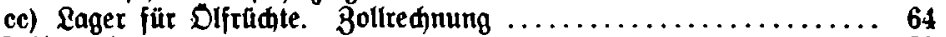

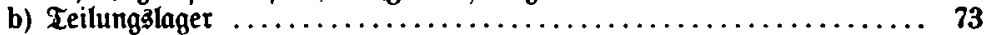

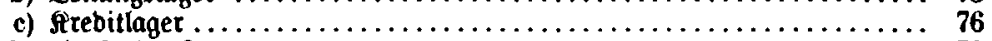

C. Fortlaufenbe fonten $\ldots \ldots \ldots \ldots \ldots \ldots \ldots \ldots \ldots \ldots \ldots \ldots \ldots \ldots \ldots$

II. Der seutfde 3olltarif.

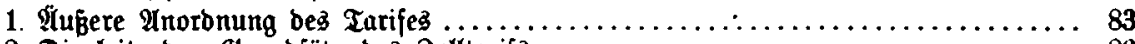

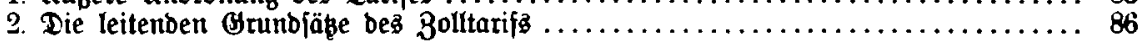

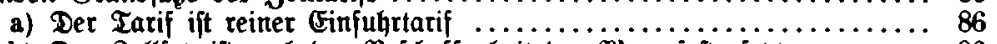

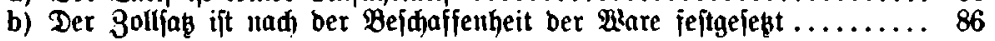


c) Die Döhe bes solles ber einzelnen

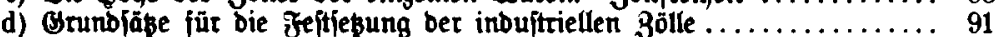

e) 2 ie witb ber zur Erthebung gelangende 3 oll bemeifen? ............ 95

\section{Die 3ollabfertigung.}

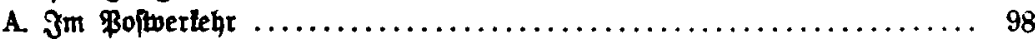

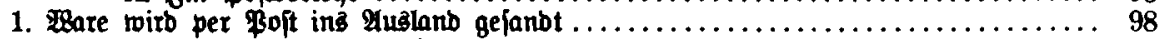

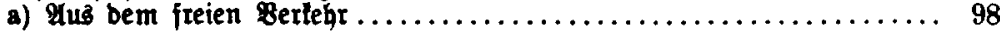

b) Qus bem gebumbenen Bertehr .......................... 105

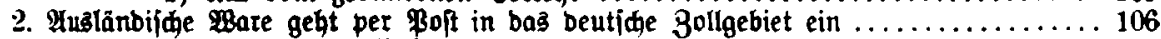

a) Bewöhntidne ßalete $\ldots \ldots \ldots \ldots \ldots \ldots \ldots \ldots \ldots \ldots \ldots \ldots \ldots, 106$

aa) Mbjertigung auf Bollvormertidein ..................... 112

bb) $\mathfrak{A b}$ fertigung als $\mathfrak{S}$ tetourmate $\ldots \ldots \ldots \ldots \ldots \ldots \ldots \ldots \ldots \ldots \ldots \ldots$

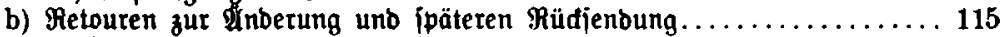

B. Im Gijenbahnverlehr .................................. 117

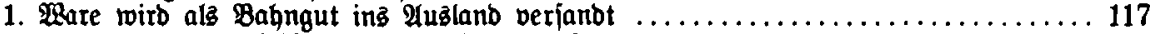

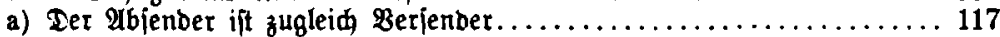

b) Der Berienber bebient fid eines Epebiteurs ald

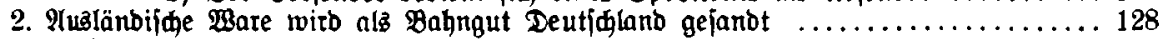
जIg Etüdgut: :

a) Tie Bollforntularitäten erlebigt bie Bahn an bet Brenze bez beutjhen

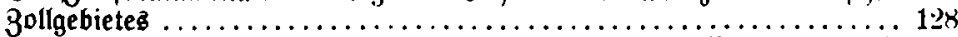

b) Die 3ollabjertigung exjolgt am Bejtimmungsonte ber \$are immerhalb

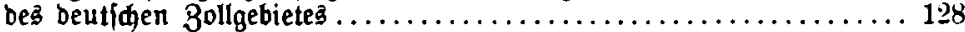

c) Die Berzollung wiro burd einen Epebiteur bejorgt $\ldots \ldots \ldots \ldots \ldots \ldots 130$

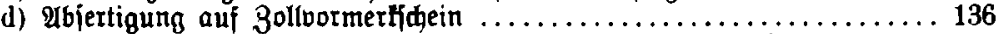

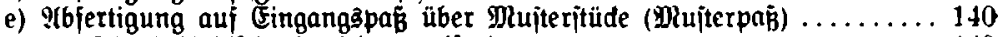

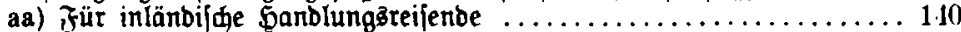

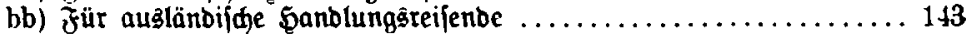

f) Einfuhr von auslänbifinen $\mathfrak{B a r e n}$ gegen 3ollhinterlegung $\ldots \ldots \ldots \ldots \ldots 14$

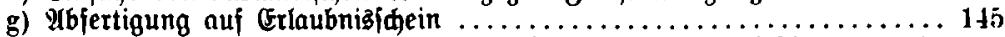

h) Ilbjertigung von Waren, bie von ber Zollbehürbe ungeniegbar gentadit

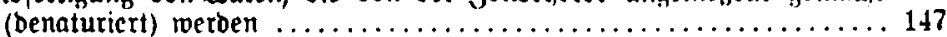

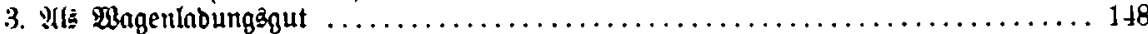

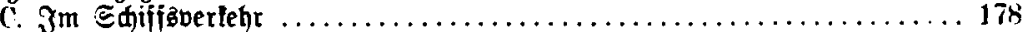

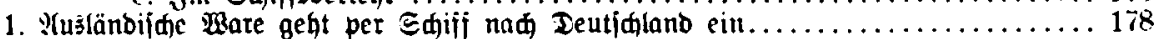

a) Der \$eiterveriand in bas beutide Zollgebiet erjolgt als Eammelgut 178

b) Der Beiterverjand in bas beuti币e 3ollgebiet erjolgt als Etüđgut ..... 188

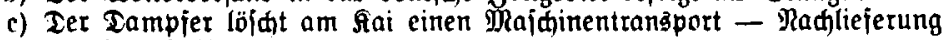
cinzelner Teile ................................... 200

d) Der 2 eiterberfand in bas beutidje 3ollgebiet erjolgt zu wajier ......203 aa) Ter Dampfer löjht im Strom - Edjutenabnahme .......... 203

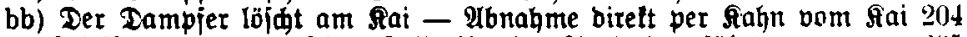

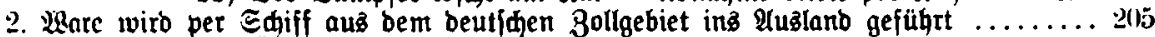

a) Dic thberiührung vom Büterbahnhof zum ftai erfolgt burd bie bajenbahn 222

b) Die ulberjührung bom Büterbahnhof zum fai erjolgt burd Befpann 232

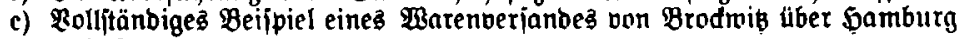
nad Enntos 